\title{
Zur Mikromorphologie, Systematik und Entwicklung der rezenten und fossilen Lößböden
}

\author{
Von W. L. Kubiena, Hamburg-Reinbek \\ Mit 4 Tafeln (II-V) und siner schematischen Darstellung
}

$\mathrm{R}$ és u mé. Les investigations dans le terrain et dans le laboratoire avec des coupes minces de sols récents et de fossils sur loess ont données la possibilité de déterminer l'existence de types caractéristiques d'assemblage microscopique, et de reconstruire un nombre de séries de dévelop.pement qui dépendent de certains districts de distribution. Pour faciliter la diagnose de différentes formations de sol sur loess, dont la détermination seulement par le profil où par l'analyse chimique a été difficile, comme la braunerde et parabraunerde, le lessivé, pseudo-gley et des formations czernozemiques, on a surtout fait attention aux caractères micromorphologiques qui sont particulairement utiles aux fins taxonomiques. Dans la région de transition entre la zône de la steppe, on trouve un sol noire particulier avec d'apparence czernozemique mais non formé sur loess brut, mais sur „parabraunerde“ très décomposée. C'est une formation tirsoide avec une série de caractères micromorphologiques particuliers qui permettent une distinction facile entre le vrai czernozem et le czernozem dégradé.

$\mathrm{Summary}$. Field and fabric investigations within thin sections of recent and fossil soil formations on loess gave the possibility to determine the existence of definite microscopic fabric types and to establich definite series of soil development which are dependant upon definite areas of distribution. In oder to facilitate the diagnosis of some soil formations on loess which are difficult to distinguish by profile and by chemical investigation, such as braunerde, parabraunerde, lessivé, pseudo-gley and chernozem-like formations, special attention was given to the well characterizing micromorphological properties, which are particularly suitable for taxonomic purposes. In the transition area between the humid and the steppe zone, a particular black soil with somewhat chernozem-like appearance occurs which is not formed on rawloess like the chernozem, but on highly weathered braunerde. It is a tirsoid formation showing a series of particular micromorphological characters which allow an casy distinction from the true and degraded chernozem.

In den letzten Jahren hatte ich Gelegenheit, die wichtigsten Lößgebiete Deutschlands, Belgiens und die Lessivégebiete Südenglands, die wahrscheinlich weitgehend von früheren I.ößablagerungen beeinflußt sind, $\mathrm{zu}$ bereisen und Rahmenproben $\mathrm{zu}$ sammeln. Von diesen (175 Einzelproben) wurden Dünnschliffe angefertigt und die Präparate mikromorphologisch untersucht; die vorliegende erste Mitteilung hat den Zweck, über die Ergebnisse in kurzer, zusammengefaßter Form zu berichten. Für die Auswertung und Typisierung wurden auch in früherer Zeit gewonnene Proben aus Ósterreich, der Tschechoslowakei, den U.S.A. und aus Frankreich benützt. Die Untersuchungen geben Gelegenheit, die Systematik der Lößböden zu überprüfen und zu ergänzen; ferner wurden bereits im Felde Studien über die rezente und fossile Bodenentwicklung durchgeführt, woraus sich eine deutliche Verschiedenheit nach Bodenprovinzen feststellen ließ. Bezüglich der Mikromorphologie wurden bei der DEUQUA-Tagung in Lauffen die wichtigsten Gefügetypen in Form von Farbdiapositiven vorgeführt. Von diesen kann in dieser Mitteilung nur ein kleiner Teil in Schwarz-Weiß-Reproduktion beigegeben werden.

Der Löß erweist sich für mikroskopische Studien der Bodenentwicklung als ein außerordentlich günstiges Ausgangsmaterial. Der Rohlöß ist wie ein unbeschriebenes Blatt, auf dem sich die kleinsten Varianten der Umwelteinflïsse mit außerordentlicher Klarheit einzeichnen, und die Mannigfaltigkeit der Gefügebildung zeigt, daß die Möglichkeiten der Auswertung noch lange nicht erschöpft sind. In diesem Sinne will auch diese Abhandlung nur der ersten Orientierung dienen. Mit besonderer Freude und Anteilnahme konnte ich feststellen, daß inzwischen auch von anderen Fachkollegen mikromorphologische Studien über Lößböden unabhängig voneinander und von mir durchgeführt wur- 
den, die zu gleichen oder weitreichend ähnlichen Ergebnissen gelangt sind (s. H. Altemüller, O. Meyer, B. Ulrich und K. Zimmermann). Diese gelangten mir erst nach Fertigstellung dieser Mitteilung zur Kenntnis.

\section{A. Vergleichende Mikromorphologie der wichtigsten Bodentypen}

\section{Arktische Boden provinz}

1. Der a rktische Rohboden ( $\mathrm{R}$ åmark). Bezüglich der Bildungen der arktischen Bodenprovinz muß vieles hypothetisch bleiben, da kalkreiche Lösse in der heutigen Arktis für ein Studium der rezenten Bodenentwicklung nicht vorliegen. Aus den Erfahrungen mit silikatischem Ausgangsmaterial kann jedoch geschlossen werden, daß der Rohlöß in der Hocharktis Klimax gewesen sein mußte und daß sich der Boden in der Tundrazone nicht über einen AC-Bodentypus hinausentwickelt haben konnte. Daß der arktische Lößrohboden eine Bildung der Kältewüste war, geht schon aus seiner Kalkverteilung hervor. Ein kapillar hochleitfähiges System wie der Löß entwickelt in warmen Trockengebieten einen starken Kapillarzug an die Bodenoberfläche oder bereits an die Oberflächen von Bodenspalten, die zu einer ziemlich raschen Ausbildung von Verdichtungen und Kalkkrusten führt. Solche rezente Krustenbildungen können in allen Stadien ihrer Entwicklung besonders schön in dichten Feinsandmergeln der Küstenzone der Kanarischen Inseln, vor allem auf Teneriffa und Gran Canaria beobachtet werden. Auf ein Trockenklima deutet andererseits die geringe Kalkwanderung nach unten.

2. Das Tundra-Anmoor. Daß gute fossile Anmoorbildungen in den kaltzeitlichen Ablagerungen nicht vorliegen, sondern lediglich Fließerden mit ausgeprägtem Gleycharakter, deutet auf humusarme hocharktische Formen. In der heutigen Tundrazone sind humusreiche Tundra-Anmoore selbst auf silikatischem Ausgangsmaterial nicht selten.

3. Die Tundra-Pararendsina. Daß eine von den Tschernosemen und Pseudo-Tschernosemen (Prärie-Erden, s. Absatz 9) unterscheidbare terrestrische TundraParasendsina vorhanden gewesen sein mußte, geht aus dem Parallelvorkommen der auf silikatischem Ausgangsmaterial gebildeten Tundraranker in der Jetztzeit hervor. Es sind dies Humusböden, in denen schon klimatisch, in der Regel auch durch das Vorhandensein einer permanenten Gefrornisschicht, die Entwicklung einer Regenwurmfauna und damit die Bildung von Tschernosem-Mull unmöglich ist. Nach den Erfahrungen mit Tundrarankern muß die Humusform der Tundra-Pararendsinen entweder Kalkmoder oder mullartiger Rendsinamoder mit gut erhaltener Kleintierlosung gewesen $\operatorname{sein}^{1}$ ). Sie mußte sich ferner durch eine weit geringere Kalkauswaschung und Bildung von $\mathrm{Ca}-$ Horizonten (vielleicht auch durch ein Fehlen von solchen) als bei den AC-Boden der Bodenprovinzen II und III charakterisiert haben. Eine solche fossile Humusbildung war mir bisher noch nicht zugänglich; es ist aber möglich, daß sie unter den vereinzelten, meist verlagerten Humusresten, die mikromorphologisch noch nicht untersucht wurden, gefunden wird.

\section{Balt is che Boden provinz}

Im feuchttemperierten Klima geht die Bodenentwicklung vom Rohbodenstadium (Syrosem) gleichfalls über ein Pararendsina-Stadium, das hier aber nur eine kurzdauernde UUbergangsbildung ist. Die terrestrische Hauptform ist die Parabraunerde, die - zunächst ungebleicht oder wenig gebleicht - bald in eine gebleichte Parabraunerde oder Lessivé übergeht.

1) Es ist anzunehmen, daß sich die Tundra-Pararendsina deshalb so wenig erhalten hat, weil der Tundra-Gürtel verhältnismäßig schmal gewesen zu sein scheint und der arktische AC-Boden bald eine Umprägung zu höher entwickelten Böden erfahren hat. 
4. Die Pararendsin a. Diese hat im Profil das Aussehen eines seichtgründigen Tschernosems, hat aber immer nur beschränkte Ausbreitung. Man trifft sie immer in Gesellschaft von Braunerden und Parabraunerden an. Sie findet sich, da sie kurzlebig und darum verhältnismäßig selten ist, am leichtesten an Standorten, wo durch Erdbauten die frühere Bodendecke entfernt worden war und neue Bodenentwicklung eingesetzt hatte. Sie ist in dieser Bodenprovinz immer eine Mullrendsina.

5. Die Braunerde. Dieser Typus ist in der baltischen Bodenprovinz auf den meisten anderen Ausgangsmaterialien die herrschende Bodenbildung, sonderbarerweise aber $\mathrm{nicht}$ a uf $\mathrm{dem} \mathrm{Löß.} \mathrm{Das} \mathrm{Braunerdegefüge} \mathrm{zeigt} \mathrm{sich} \mathrm{durch} \mathrm{eine} \mathrm{mehr} \mathrm{oder}$ weniger starke, doch stets deutliche Flockung aller Schlämmstoffe, die besonders stark an den Eisenhydroxydgelen in Erscheinung tritt. Die Masse bildet dadurch ein besonders hohlraumreiches Schwammgefüge, das sich aus schorfig-krümeligen, miteinander leicht verbundenen Aggregaten zusammensetzt (vgl. Tafel II, 2). Auf Löß tritt die gewöhnliche mitteleuropäische Braunerde in der Regel in den wärmeren und trockeneren Teilen der Provinz auf, so als fossiler Boden im Rheingau (s. Tafel II, 3). Der Profilaufbau ist in der Regel $\mathrm{A}(\mathrm{B}) \mathrm{Ca}$, wobei Ca-Horizonte zumeist gut ausgebildet sind.

Die Muttergesteinsgruppe, die selten zu Braunerdebildung führt, sondern auf der sich Bodendecken mit zumeist stark ausgeprägten Braunlehmgefügen bilden, sind die Kalkgesteine. Die aus diesen als reife Bodenbildung hervorgehende Terra fusca (KalksteinBraunlehm) zeigt besonders im (B)-Horizont mikromorphologisch eine dichte, fast ungekrümelte, zumeist grellockergelb gefärbte Grundmasse, in der das Eisenhydroxyd hochbeweglich und diffus zerteilt ist. Scheidet sich an irgend einer Stelle Eisenhydroxyd in Gelform aus, so wird das Diffusionsgleichgewicht gestört, und die wanderungsfähigen Teilchen bewegen sich von allen Seiten zur Ausscheidungsstelle und bilden eine runde Konkretion mit glatter Oberfläche und von tiefbrauner Farbe. Dieses Braunlehmgefüge der Terra fusca bildet zu einem Braunerdegefüge mit guter Flockung den stärksten Gegensatz. Es wurde hier angeführt, da sich ähnliche Teilgefüge in den Böden der Lessivégruppe (Absätze 6-10) vorfinden.

6. Die Parabraunerde. Dieser A(B)CaC-Boden ist äußerlich der Braunerde ähnlich, und die noch ungebleichten und schwach gebleichten Formen sind bisher auch immer als Braunerde angesprochen worden. Der Unterschied wird aber im Dünnschliff sofort sichtbar. Wir sehen ein Braunerde-Grundgefüge, während sich in den wegsamen Gefügeteilen (Leitbahnen) auffallende wandständige Anlagerungen oder die Hohlräume völlig ausfüllende Schlämmstoffkomplexe von Braunlehmcharakter und von leuchtend ockergelber (Munsell 2,5 YR 7/8) bis ockerbrauner (7,5 YR 5/6) Farben feststellen lassen. Wir sprechen von einem Braunerde-Teilplasma ${ }^{2}$ ) und einem Braunlehm-Teilplasma, wobei jenes unbeweglich ist und am Orte bleibt, dieses aber hochbeweglich ist, darum in die Leitbahnen des Gefügekörpers abströmt. Das gleiche Gefüge sehen wir weit stärker ausgebildet im B-Horizont der gebleichten Parabraunerde. Während die ungebleichte Parabraunerde noch keinen Bleichhorizont aufweist, zeigt jene bereits einen $\mathrm{A}_{\mathrm{e}}$-Horizont. Aber auch in der ungebleichten Form zeigt sich bei dem Braunlehm-Teilplasma bereits die Tendenz einer Abwärtsbewegung im Unterschied zu dem zu großem Teile ungerichteten Braunlehmplasma im Pseudogley.

Die ungebleichte Parabraunerde ist die Entwicklungsvorstufe zur gebleichten Form. Sie ist zufolge der starken Beweglichkeit des Braunlehm-Teilplasmas eine kurzlebige Form. Sie ist entwicklungskundlich wichtig, denn sie ist das Glied zwischen Pararendsina und gebleichter Parabraunerde. Nahe dem Braunerdeverbreitungsgebiet oder an

2) Unter Gefügeplasma ist die Gesamtheit der Schlämmstoffe und löslichen Salze gemeint, d. h. der feindispersen, hochaktiven, umwandlungsfähigen Anteile eines Gefüges gegenüber dem starren, grobdispersen Gefügeskelett. Aus dem Wechselverhältnis beider ergeben sich bestimmte Typen von Elementargefüge (s. KuBIENA 1938). 
austrocknenden Standorten ist eine Tendenz der Umwandlung von ParabraunerdeGefügen in Braunerdegefüge durch zunehmende Flockung und Vererdung des Braunlehm-Teilplasmas festzustellen.

7. Die gebleichte Parabraunerde (Lessivé). Dieser 'Typus mit dem Profilaufbau $\mathrm{AA}_{\mathrm{e}} \mathrm{BCaC}$ ist in der baltischen Bodenprovinz auf Löß die führende terrestrische Bildung. Äußerlich einer podsoligen Braunerde ähnlich, unterscheidet sie sich von dieser jedoch in auffallender Weise durch das Gefügebild. Im B-Horizont zeigt sie ein ähnliches Bild wie der (B)-Horizont der noch ungebleichten Parabraunerde, doch sind die Leitbahnen zahlreicher, stärker entwickelt, die Abwärtsbewegung des Braunlehmplasmas im ganzen Profil stärker ausgeprägt. Mitunter trifft man außerordentlich breite Leitbahnen (von etwa 0,5-300 mm Durchmesser) an, die völlig mit dichtem Fließplasma erfüllt sind (Tafel II, 3; IV, 2, 3). Die Leitbahnfüllungen erscheinen mit freiem Auge schmutzig-ockergrau (5 YR 6/4) bis schokoladenfarbig (5 YR 4/3), im Dünnschliff leuchtend ockergelb (10 YR 7/8) bis ockerbraun (5 YR 5/6) gefärbt. A und $\mathrm{A}_{\mathrm{e}^{-}}$ Horizont zeigen stets eine deutliche Verarmung an Schlämmstoffen, doch wird der $A_{e}$ nie so bleich wie bei einem Podsol, weil immer ein erheblicher Teil des geflockten Braunerde-Teilplasmas in ihm erhalten bleibt. Als besonderes Merkmal zeigt sich in beiden A-Horizonten das Auftreten von tiefbraunen Konkretionen von Eisenhydroxyd (Tafel IV, 1, 2). Sie sind zumeist unregelmäßig geformt und nicht so glatt, wie in dichten Braunlehmgefügen, sind diesen aber weitgehend analog. Es ist anzunehmen, daß ihre Bildung in der Entwicklungsvorstufe der ungebleichten Parabraunerde erfolgt ist, doch kommen $\mathrm{A}_{\mathrm{e}}$-Horizonte vor, die auch andere Parabraunerde- oder Pseudogley-Merkmale haben, wie überhaupt zwischen den Typen je nach dem Standort, diesen scharf charakterisierend, alle Übergangsbildungen gefunden werden können. Die Konkretionen sind mannigfaltig geformte, dicht gefügte Eisen-(Mangan-)Flecken. Sie können auch in den Leitbahnfüllungen der B-Horizonte (Tafel IV, 2, 3) entstehen. Die Humusform ist fast immer Mull. Nur dort wo sich der Lessivé dem Podsoltypus nähert, entstehen besonders im Nadelwald dystrophe Humusformen.

Die Kalkanreicherungshorizonte zeigen wie die B-Horizonte das Einfließen von Braunlehmplasma in seine Leitbahnen. Solches Fließplasma ist bereits auch in Ca-Horizonten ungebleichter Parabraunerden festzustellen. Die Kalkhorizonte sind jedoch häufig weniger stark ausgeprägt als bei den Tschernosemen und den Kalkbraunerden auf Löß und können vollständig fehlen.

Die Bezeichnung Lessivé (sol lessivé) wurde von der französischen Bodenkunde geprägt. Die Bodenbildung war wohl in anderen Ländern bekannt (U.S.A., Deutschland), ist aber nicht als eigener Typus beschrieben und benannt worden. Leider sind in den ursprünglichen Lessivé-Begriff auch Böden der feuchten Tropen einbezogen worden. Wenn aber Bodenbildungen als charakteristische Produkte und Anzeiger bestimmter Umweltbedingungen gewertet werden sollen, so müssen die Begriffe der Bodensystematik stets auch bodengeographisch richtig sein und bei Auftreten krasser Widersprüche rektifiziert und anders zu fassen versucht werden. So ergibt sich auch die Notwendigkeit der Einengung des Lessivé-Begriffes auf die Formen des feuchtgemäßigten Klimas. Diese ist auf Grund ihrer Mikromorphologie möglich. Die mitteleuropäischen Lessivés zeigen immer das Auftreten des Braunerdegrundgefüges und des Braunlehm-Teilplasmas in Leitbahnen, während die tropischen und subtropischen Formen als gebleichte $\mathrm{Braunlehme}$ in allen Teilen des B-Horizontes ein Gefügeplasma von Braunlehmcharakter haben, auch in der Grundmasse (s. Kubiens 1953, p. 270). ${ }^{3}$ )

$\left.{ }^{3}\right)$ Auch die sogenannte gebleichte Terra fusca ist keine gebleichte Parabraunerde, denn im (B)-Horizont zeigt sich ein unverändertes Braunlehmgefüge. 
Wenn man darum von Lessivé auf Löß spricht, so kann damit nur der mitteleuropäische Lessivé gemeint sein. Ich übernehme für diesen den von E. MückENHAusEN geprägten Namen Parabraunerde, unterscheide aber die Parabraunerde schlechtweg und die gebleichte Parabraunerde für die Lessivéform. ${ }^{4}$ ) Der aus den U.S.A. stammende Begriff des "graybrown podsolic" ist zu größtem Teile mit dem mitteleuropäischen Lessivé synonym, umfaßt aber auch auch die podsolige Braunerde RAMANN's und andere Semipodsole.

8. De r Pse udogley. Dieser. durch Staunässe entstandene, durch einen Wechsel von Durchfeuchtung und Austrocknung charakterisierte Boden zeigt im Dünnschliff neben der Einwirkung von Gerbsäuren (mitunter auch von Humussolen) und teilweiser Reduktion das Vorhandensein eines ähnlichen, leuchtend hellocker (10 YR 7/6) bis ockerbraun (7,5 YR 5/6) gefärbten Braunlehm-Teilplasmas wie bei den vorausgehenden Typen. In der Farbgebung kommen durch Humusfärbung auch schmutzig-braungraue Farbtöne vor. Dieses Braunlehmplasma ist jedoch, besonders im $\mathrm{g} /(\mathrm{B})$-Horizont, nur zum Teil in Leitbahnen gesammelt und konzentriert, es ist vielmehr vorwiegend in unregelmäßigen Flecken im Grundgefüge enthalten. Das gestaute Bodenwasser hat wohl einige, aber nie so starke und durchgreifend reduzierende Wirkung (selbst im g-Horizont nicht) wie bei den Gleyen und Stagnogleyen, so daß das Ferri-Eisen im Braunlehmplasma weitgehend erhalten bleibt. Jedoch zeigt sich in den nicht von Braunlehm-Teilplasma durchsetzten Gefügeteilen schon so gut wie kein Braunerdegrundgefüge mehr. Die nicht intensiv ocker gefärbten Partien sind hellgrau bis farblos. Das Ferri-Eisen ist hier zu großem Teile nicht reduziert, sondern in Ferri-Form verlagert worden. Hierin scheint der Hauptunterschied zwischen der echten Vergleyung und der Pseudovergleyung zu liegen. Die Verlagerung kann so erfolgen, daß das ockerfarbige Gefügeplasma als Ganzes bewegt wird oder daß es am Ort bleibt und sich nur das Eisen daraus entfernt hat. Neben den Konkretionen im g-Horizont, die jenen im $\mathrm{A}_{\mathrm{e}}$-Horizont des Lessivés ähnlich sind (s. Tafel III, 2), kommen bereits auch tiefbraune lockere unregelmäßig geformte Eisenflecke mit unscharfen Rändern vor, wie sie für die Stagnogleye typisch sind.

9. Der Pseudo-Tschernosem ( Prärie-Erde), n. f. Dieser Typus wurde bisher in der Regel als degradierter Tschernosem beschrieben, wobei jedoch immer auf die stets vorhandenen Übergänge $\mathrm{zu}$ anmoor-ähnlichen Formen oder direkt zu Anmooren hingewiesen wurde. Der Unterschied zwischen dem Pseudo-Tschernosem und dem echten Tschernosem und dessen degradierter Form tritt vor allem im Schliffbild recht deutlich in Erscheinung. Außerlich hat der Boden zumeist den Profilaufbau eines degradierten Tschernosems mit einem A-Horizont, der eine Mächtigkeit von $50-80 \mathrm{~cm}$ erreichen kann, an den sich saumförmig oder voll ausgeprägt ein (B)-Horizont anschließt

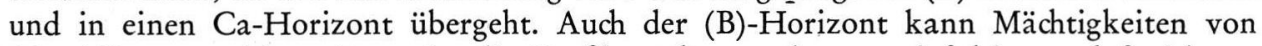
$60-100 \mathrm{~cm}$ erreichen. Sonst ist die Profilgestaltung sehr mannigfaltig, so daß sich sowohl Übergänge zu einem Parabraunerde- als auch zu einem Lessivé- oder Pseudogleyprofil feststellen lassen können. Im Unterschied zum Tschernosem ist der Boden auch im Humushorizont dicht, beim Austrocknen scharfkantig zerfallend, im feuchten Zustande auffallend schwärzlich bis schwarz, stark klebend, stark erodierbar und verschlämmbar, im trockenen Zustande steinartig verhärtend.

Der Tschernosem ist ein durch langsame chemische Verwitterung charakterisierter terrestrischer Mullboden, der sich durch stete Vertiefung des A-Horizontes unmittelbar aus dem Rohlöß entwickelt. Die degradierte verbraunte Form entsteht durch Ansatz

$\left.{ }^{4}\right)$ Ich schlug für Lessivé auch als deutsches Synonym „Brickerde“ vor (nach dem altniederländischen "bricke" = Ziegel; vgl. auch die in Frankreich, Belgien und England gebräuchlichen Bezeichnungen „terre-à-brique " und „brick earth“ für das B- und E/B-Material. Doch lasse ich diese Bezeichnung auf, da mit „Parabraunerde“ die Stellung gegenüber der Braunerde stärker zum Ausdruck kommt. 
eines braunerdeähnlichen (B)-Horizontes (vgl. Absatz 11 und 12). Der Pseudo-Tschernosem ist eine tirsoide Bildung, die auf den Unterhängen, in Mulden oder in ebener Lage vorkommt und starkverwitterte (häufig als Erosionssediment vorhandene) reife Bodenbildungen zum Ausgangsmaterial hat, die äußerlich durch braunlehmartige Merkmale auffallen. Er ist der Tirs der gemäßigten Breiten, charakterisiert durch winterfeuchtes, aber verhältnismäßig sommertrockenes Klima. Er zeigt sich vorwiegend im Ubergangsgebiet zwischen Steppen- und Feuchtzone, besonders typisch in Form des N-S-Gürtels der ehemaligen Langrassteppe mit Galeriewäldern in den U.S.A. Wie jede tirsoide Bodenbildung entsteht er auf Böden mit geringer Wasserdurchlässigkeit (der Tschernosem nimmt Wasser leicht auf), wo durch Stauung zunächst eine anmoorähnliche Humusbildung entsteht, die sich allmählich in Mull umwandelt. Im Winter und Frühjahr ist der Boden auch in späteren Entwicklungsstadien mehr oder minder stark vernäßt und wird erst im Spätfrühjahr erdig. Er krümelt dann gut, die Krümel haben aber eine auffallend geringe Stabilität, so daß er nach Regenfällen von oben her vollständig dicht verschlämmt. An der Oberfläche zeigt sich eine auffallende Kornscheidung so daß diese stellenweise mit weißen Flecken von reinem Quarzsand oder Quarzmehl bedeckt erscheint (s. KubiEna 1938, p. 179).

Mikromorphologisch zeigt der A-Horizont ein sehr hohlraumarmes Gefüge mit humoser dunkel-bräunlichgrauer, fleckiger, aber dicht zusammengedrängter Grundmasse. Von dieser scheidet sich in Flecken und Leitbahnen ein stark bewegliches BraunlehmTeilplasma ab, dessen Natur am besten in den Anlagerungen und Ausfüllungen der Leitbahnen sichtbar wird (Tafel V, 2). In der Regel zeigt es starke Teilchendoppelbrechung und Fließstrukturen und ist stärker als in den anderen Bodenbildungen mit feinzerteilten Humusstoffen angereichert. Ihre Farbe ist darum auffallend schwärzlich, im Dünnschliff bei Durchlicht dunkelgraubraun (7,5 YR 4/2) bis grauocker (7,5 YR 5/4). Zumeist ist der ganze Humushorizont und der darunterliegende (B)-Horizont mit unzähligen kleinen Leitbahnen durchsetzt (s. Tafel V, 2); doch kommt das dunkle Fließplasma auch in pseudogley-ähnlichen Flecken vor (s. Tafel V, 1). Diese stark beweglichen Fließplasmen oder Bodenteile, die von solchen stark angereichert sind, werden auch makroskopisch in auffallender Weise sichtbar: die schwärzlichen, plasmoiden, stark beweglichen Ton-Humusmassen fließen bei feuchtem Wetter in alle Hohlräume, wie Maulwurfsgänge, Wurm- und Wurzelröhren und Bodenspalten, ein und können sich ziemlich weit in den Unterboden hinabbewegen, eine Erscheinung, die bei den echten Tschernosemen fehlt.

10. Der Stag nogley (F. VogeL). Während der Pseudogley ein wechselfeuchter Boden ist, charakterisiert sich der Stagnogley durch eine das ganze Jahr hindurch andauernde Staunässe. Das Stauwasser hat hier ferner eine stark reduzierende Kraft. Aus Pseudogley oder anderen der oben genannten Typen der hydromorphen Reihe gebildete Stagnogleye zeigen, daß auch das Ferri-Eisen, das im Braunlehm-Teilplasma in peptisierter, leicht diffusibler Form vorhanden gewesen war, weitgehend reduziert ist. Hingegen treten kräftig dunkelbraun gefärbte, fast opake Flecken mit stark zerfressenen Rändern auf (Tafel V, 4), deren Bildung schon bei den nassen Pseudogleyen beginnt, bei den Stagnogleyen aber ihren Höhepunkt erreicht.

\section{Pannonische Boden provinz}

11. Der Tschernosem. Dieser Typus überwiegt als rezente Bildung im östlichen Ósterreich mit zum Teil mächtigen Humushorizonten (bis zu 1)m) und gut ausgebildeten Ca-Horizonten. Im Gegensatz zum Pseudo-Tschernosem oder der Prärie-Erde zeigt er sich im Dünnschliff nicht dicht gefügt, sondern hat ein ausgesprochenes Schwammgefüge, in dem entweder Losungspakete von Regenwürmern gut erhalten sind oder in dem sich - besonders bei degradierten Formen - mehr schorfig-krümelige Aggregate 
(ähnlich wie bei der Braunerde) aneinander gefügt haben. Jedenfalls erscheint die humose Grundmasse gut geflockt, die Hohlräume sind vollkommen frei von Schlämmstoffeinlagerungen, ein Braunlehm-Teilplasma wird nirgends sichtbar. Die chemische Verwitterung ist gehemmt, viele im Feuchtklima leicht zersetzbare Mineralien wie Biotite sind vielfach gut erhalten. Die Humusbildung ist ein rein terrestrischer Mull, in dem keine Anklänge an Anmoorhumus festzustellen sind, eine Kornscheidung an der Bodenoberfläche durch den Regen tritt nicht ein.

12. Derdegradierte Tschernosem. Diese verbraunte Form ist als rezente Bildung an der Grenze des Steppengebietes gegen die Waldzone in trockenen Hügellagen häufig zu finden. Der zumeist wenig mächtige saumartige (B)-Horizont zeigt im Mikroskop ein ausgesprochenes Braunerdegefüge mit guter Flockung und zahlreichen, von Schlämmstoffeinlagerungen völlig freien Hohlraumbildungen. Der Boden zeigt gute Wasserdurchlässigkeit, geringe Erodierbarkeit und eine hohe Krümelstabilität. Der Boden ist in den Ubergangsgebieten eine Klimaxform, leitet aber mikromorphologisch zu den Kalkbraunerden auf Löß über.

13. $\mathrm{D}$ i e $\mathrm{Br}$ a u e r de. In der pannonischen Bodenprovinz kommt diese nur als rezente Bildung vor und ist für die klimatisch feuchten Lagen der Steppe, ferner für die Berg- und Hügellagen der an die Steppe grenzenden Waldgebiete charakteristisch. Sie zeigt, wie bereits in Absatz 5 ausgeführt, ein gut geflocktes hohlraumreiches Schwammgefüge. Die typischsten Vorkommen fand ich bei Neubau a. d. Ostbahn in Niederösterreich.

14. Tirsifizierte und rubefizie rte Böden. Unter den stark verwitterten fossilen Böden im pannonischen Raum zeigen sich nicht Braunerden, sondern stark braunlehmartige "Parabraunerden“, die mehr oder minder stark rubefiziert sind. Auf ihnen sind tirsoide Humusformen bis echte 'Tirsbildungen nicht selten, die ebenso wie die Rubefizierung auf ein wechselfeuchtes Klima hindeuten. Die Art der Bodenbildung zeigt bereits einen stark mediterranen Einschlag. ${ }^{5}$ )

\section{B. Zur allgemeinen Genese der Böden der Lessivé-Gruppe}

15. Problemstellung. Die Böden der hydromorphen Reihe der mitteleuropäischen Lessivé-Gruppe, d. i. die Parabraunerde, gebleichte Parabraunerde, Pseudo-Tschernosem, Pseudogley und Stagnogley, sind Böden eines Braunerdeklimas. Parabraunerde, Lessivé und Pseudo-Tschernosem zeigen dies durch das Vorhandensein eines Braunerdegrundgefüges an, das sich beim Pseudogley und Stagnogley durch den Einfluß der Staunässe zu großem Teile verloren hat. An Standorten, an denen die Klimaeinwirkung im Sinne von Braunerdebildung besonders stark in Erscheinung tritt, kann auch das Braunlehm-Teilplasma zu großem Teile zu Braunerdegefügen umgeprägt werden. Allen Normalbildungen gemeinsam ist aber ein stark ausgeprägtes Braunlehm-Teilplasma, das von entscheidendem Einfluß auf ihre Dynamik und besondere Eigenart ist. Dieses ist auch beim Stagnogley noch deutlich vorhanden, wenn auch die Ferrihydroxyde in ihm weitgehend reduziert sind. Da Braunlehmgefüge nach den bisherigen Erfahrungen Produkte eines feucht-subtropischen bis tropischen Klimas sind und sich bisher im feuchttemperierten Klima nur als Fossil- oder Reliktbildungen vorgefunden hatten, ist vor allem die Bildung oder Herkunft des Braunlehm-Teilplasmas in den Böden der mitteleuropäischen Lessivégruppe $\mathrm{zu}$ erklären.

16. Hypothesen zur Herkunft des Braunlehm-Teilplasmas. Hierzu gibt sich im wesentlichen die Möglichkeit zu folgenden Annahmen:

${ }^{5}$ ) Auf den an die DEUQUA-Tagung in Lauffen anschließenden Exkursionen wurden vom Verfasser in Nied.-Österrerich und Burgenland von allen typischen Lößprofilen Rahmenproben dieser. Böden für Dünnschliffe genommen. Die Mitteilung der Ergebnisse ihrer Untersuchung wird in einer besonderen Abhandlung erfolgen. 
a) Das Braunlehm-Teilplasma stammt aus vorpleistozänen tropischen bis sub-tropischen Verwitterungen und war bereits im Rohlöß enthalten. Mit der Entkalkung des Lösses wurde es aktiv, konzentrierte sich und wirkte auf das durch Verwitterung in situ entstandene Braunlehmplasma wie ein Schutzkolloid, indem es auch auf dieses eine weitgehend zerteilende Wirkung ausübte.

b) Auch die im Braunlehm- Teilplasma als wirksamstes Schutzkolloid autretende kolloidale Kieselsäure entsteht im feuchtgemäßigten Klima im Laufe der Bodenbildung in situ, wobei die für eine intensive chemische Verwitterung günstige Klimavariante, die starke Durchfeuchtung und das hohe Festhaltevermögen des Bodens für entstandene Kieselsäuresole (zufolge seiner hohen Kapillarität), von entscheidendem Einfluß sind.

c) Als Ausgangsmaterial für den Rohlöß treten sowohl Silikatschotter als auch Kalkschotter auf. Silikatgesteine liefern im Braunerdegebiet Braunerde, Kalkgesteine liefern Terra fusca, die durch ein in der Regel besonders stark ausgebildetes Braunlehmgefüge charakterisiert ist (s. Absatz 5). Neben der direkten Verwitterung in situ kommen noch die auf beiden Muttergesteinsarten früher entstandenen, im Löß stets bereits vorgebildet enthaltenen Reste alter Bodendecken. Von dem Braunlehmanteil geht, wie bei a) angeführt, eine Schutzkolloidwirkung auf den Braunerdeanteil aus.

17. Zur Terra-fusca-Hypothese. Obwohl wahrscheinlich alle oben angeführten Einflüsse zusammen wirken, scheint mir auf der Terra-fusca-Hypothese das Schwergewicht zu liegen. Parabraunerden finden sich nicht nur auf Löß, sondern auch auf Moränen und Schottern, besonders wenn sie reich an Kalksteingeröll sind. Sie finden sich aber niemals auf metamorphem und Eruptiv-Gestein. Aus den Alpen kommend, sieht man auf den anstehenden Kalkgesteinen typische Terra-fusca-Profile, die in allen Gefügeteilen ein reines Braunlehmgefüge zeigen (zuweilen mit einer von der Bodenoberfläche ausgehenden leichten braunen Vorerdung, die aber nicht sehr in die Tiefe geht). Auch auf Moränen und Schottern mit hoher Kalkführung kann man vielfach noch Terra-fusca sehen. Auf den Moränen und Schottern mit gemischter Geröllführung sieht man jedoch Bodenbildungen, die zwar äußerlich einer Terra-fusca ähnlich sind, im Dünnschliff aber deutlich das Gefüge einer Parabraunerde zeigen. Dieses läßt sich auch bei fossilen Bodendecken feststellen. So hat auch der mir von F. WEIDENBACH vorgeführte, in Tafel IV, Abb. 4, in einem Dünnschliff wiedergegebene fossile, auf jüngerem Deckenschotter gebildete und von Rißmoränen überdeckte und dadurch gut datierte Boden aus der Umgebung von Ulm von deutlichem Parabraunerde-Charakter äußerlich ein weitgehend terra-fusca-ähnliches Aussehen. Weitgehend gestörte und zerteilte Reste solcher Bodendecken sind häufig im Inneren der Moränen und Schotter enthalten und werden mit den unverwitterten Gesteinsresten zum Ausgangsmaterial für die Lößbildung. Durch die Verwitterung des Lößes geht auch sein Kalk in Lösung und gibt die in ihm enthaltenen unlöslichen Schlämmstoffrückstände frei. Daß der Rohlöß und die echte Schwarzerde (nicht die hochgradig verwitterte Pseudoschwarzerde) wenig von dem Braunlehm-Teilplasma erkennen läßt, ist eine Erscheinung, die eine Parallele in dem Gegensatz zwischen der noch kalkreichen Mullrendsina und der vollausgebildeten, durch hohe Aktivierung und Konzentrierung der Schlämmstoffe charakterisierte Terra-fusca findet.

Das Braunlehm-Teilplasma in der Parabraunerde neigt ebenso wie das Gefügeplasma der Terra fusca stark zu Rubefizierung, die in typischem Mediterran-Klima zu Terra-rossa-Bildungen führt. Leichte Rubefizierung an einzelnen fossilen Böden wurde bereits im Rheingau beobachtet (vgl. die unmittelbar über dem oberolizänen Süßwassermergel hangende Parabraunerdeschicht im Hohlweg von Erbbach).

Die Reisen in den deutschen Lößgebieten wurden mit Unterstützung des Consejo Superior de Investigaciones Científicas in Madrid durchgeführt, wofür ich diesem und 


\section{Die wichtigsten Entwicklungstendenzen der rezenten und fossilen Lößboden}

in verschiedenen Bodenprovinzen

I. Arktische Bodenprovinz

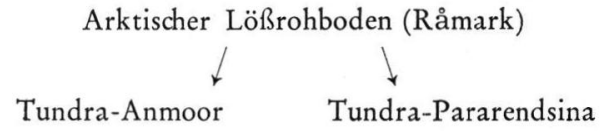

II. Baltische Bodenprovinz

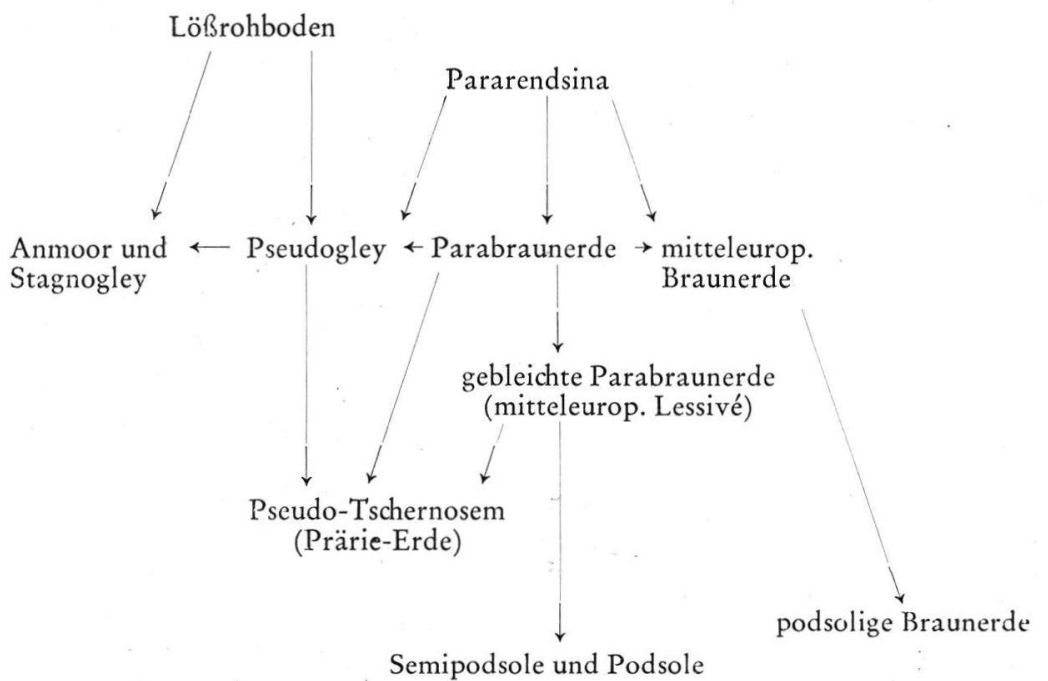

III. Pannonische Bodenprovinz

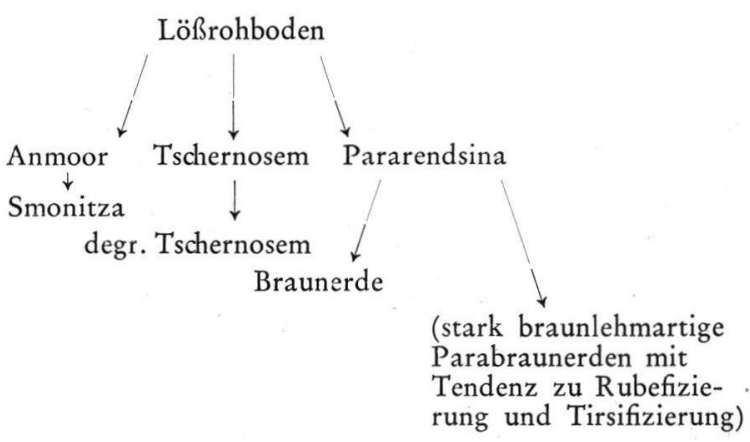


seinem Generalsekretär Herrn Prof. Dr. J. Ma Álbared , bestens danke. Ich danke auch herzlich allen jenen Herrn Kollegen und Freunden, die mich in den einzelnen Gebieten durch Sonderführungen und Exkursionen zur Entnahme der Rahmenproben in freundlichster Weise unterstützt haben.

\section{Zusammenf assung}

1. Freilandstudien und Gefügeuntersuchungen an Dünnschliffen verschiedener rezenter und fossiler Bodenbildungen auf Löß ermöglichten die mikromorphologische Definierung bestimmter Formen und die Aufstellung von Entwicklungsreihen, die in Abhängigkeit zu bestimmten Bodenverbreitungsgebieten (Bodenprovinzen) steht.

2. Als solche Entwicklungsgebiete wurden, nach dem vorhandenen Probematerial, vorläufig eine arktische, baltische und pannonische Bodenprovinz ausgeschieden, wobei die letzte bei fossilen Bodenbildungen eine feuchte Variante mit deutlich mediterranem Einschlag zeigt, die wahrscheinlich, sobald die Untersuchungsergebnisse bezüglich der inzwischen aufgesammelten Rahmenproben dieses Gebietes in Osterreich vorliegen, für die interglazialen Bodenbildungen eine neue, selbständige Bodenprovinz notwendig machen wird.

3. Die Grenzen der Bodenprovinzen in den Interglazialzeiten scheinen trotz gewisser Parallelen mit den heutigen nicht ganz übereinzustimmen. Besonders starke Divergenzen zeigen sich im Pannonischen Raum durch einen von den heutigen Böden verschiedenen, stark braunlehmartigen Charakter der interglazialen Bodenbildungen, besonders jenen des Altpleistozäns, mit einer starken Tendenz zu Rubefizierung und Tirsifizierung bis $\mathrm{zu}$ echter Tirsbildung, die ein warmes, aber weit humideres, ausgesprochen wechselfeuchtes Klima anzeigen.

4. Zur leichteren Diagnostizierung äußerlich ähnlich aussehender, durch chemische Analysen nicht unterscheidbarer Bodentypen, wie der Braunerde, Parabraunerde, des mitteleuropäischen Lessivés (gebleichte Parabraunerde mit schwarzerdeähnlichen Typen), wurden die stark charakterisierenden mikromorphologischen Merkmal besonders herausgestellt.

5. Als neuer Bodentypus, der nicht wie der Tschernosem auf Rohlöß, sondern sekundär auf hochverwitterter Parabraunerde als tirsoide Bodenbildung auftritt, wurde der Pseudo-Tschernosem (Prärie-Erde) beschrieben und seine Merkmale, die sich wieder in seiner Mikromorphologie besonders stark ausprägen, denen des echten Tschernosems und des degradierten Tschernosems gegeniibergestellt.

\section{$\mathrm{Schrift} \mathrm{tum}$}

Altemüller, H. J., 1955: Gefügeuntersuchungen an verschiedenen Löß-Bodentypen mit Hilfe der Dünnschliff-Methode. - Vortrag an der Jahrestagung d. Deutschen Bodenkdl. Ges. in Göttingen.

BrunNacker, K., 1954: Löß und diluviale Bodenbildungen in Südbayern. - Eiszeitalter und Gegenwart, 4/5, 83-86. - - 1955: Würmeiszeitlicher Löß und fossile Böden in Mainfranken. - Geologica Bavarica 25, 22-38.

Dudal, R., 1953: Etude morphologique et génétique d'une sequence de sols sur limon loessiqueExtrait de l'Agriculture 1. 121 (dortselbst weiteres Lessivé-Schrifttum).

Fink, G. \& Majadan, H., 1954: Zur Gliederung der pleistozänen Terrassen des Wiener Raumes. - Jb. geol. B.A. Wien 97, 2.

Freising, H., 1949: Lösse, Fließerden und Wanderschutt im nördlichen Württemberg. - Diss. TH. Stuttgart. - - 1951: Neue Ergebnisse der Lößforschung im nördlichen Württemberg. - Jh. geol. Abt. württ. statist. L.-Amt 1, 54-59.

Kubiena, W., 1938: Micropedology, Ames. - - 1948: Entwicklungslehre des Bodens, Wien. - 1953: Bestimmungsbuch und Systematik der Böden Europas. Stuttgart. 
LaAtsch, W., 1934: Die Bodentypen um Halle (Saale) und ihre postdiluviale Entwicklung. Jb. d. Halle'schen Verb. f. d. Erf. d. mitteldtsch. Bodenschätze u. ihrer Verwendung 13, 57.

Merer, O., 1935: Böden des Leinegrabens und Möglichkeiten zu ihrer zeitl. Einordnung. - Vortrag und Diskussionsbemerkungen an d. Jahrestagung d. D.B.G. in Göttingen.

Mǘckenhausen, E., 1955: Entwurf einer Systematik der Böden Deutschlands.

Ostendorff, E., 1954: Fossile Schwarzerden und Waldböden. - Z. f. Pflanzenern., Düng. u. Bodenk. 65, 62-79.

Schönhals, E., 1950: Uber einige wichtige Lößprofile und begrabene Böden im Rheingau. Notizbl. hess. L.-Amt Bodenforschung, Wiesbaden, 6, 244-591. - - 1951: Über fossile Böden im nichtvereisten Gebiet. - Eiszeitalter und Gegenwart 1, 109-30.

Schroeder, D., 1953: Untersuchungen über Verwitterung und Bodenbildung an Lößprofilen. Hannover.

Swineford, A. \& Frye, J. C., 1955: Petrographic Comparison of some Loess Samples from Western Europe with Kansas Loess. - Journ. Sedim. Petrology 25, 3-23.

Ulrich, B., 1955: Fossile Bodenbildungen aus dem Pleistozän und Postglazial (Braunlehm, Sol lessivé und Podsol). - Vortrag und Diskussionsbemerkungen a. d. Jahrestagung d. D.B.G. in Göttingen.

Weidfinbach, F., 1952: Gedanken zur Lössfrage. - Eiszeitalter und Gegenwart 2, 25.

Manuskr eingeg.: 13. 12. 1955.

Anschrift d. Verf.: Prof. Dr. W. Kubiena, Hamburg-Reinbek, Bundesforschungsanstalt für Forstund Holzwirtschaft, Abt. f. Bodenkunde, Schloß.

\section{Erklärung der Abbildungen}

Alle Reproduktionen zeigen eine Vergrößerung von ca. Sofach.

\section{Tafel II}

Abb. 1. Dünnschliff aus dem (B)-Horizont einer Terra fusca, südl. Wiener Wald. Dichte ungefleckte Grundmasse mit Braunlehmgefüge von leuchtend ockergelber Farbe (Munssell 10 YR 7/8), in der peptisierte Ferrihydroxyde in gleichmäßiger, diffuser Verteilung vorliegen und in welche Mineralkörner (praktisch nur Quarze) porphyrähnlich eingebettet sind. Rechts oben der Rand einer Eisenkonkretion von der für Braunlehmgefüge typischen Form. Diese zeigt scharfe Umgrenzung und eine dunkelsepiabraune Farbe (5 YR 3/2).

Abb. 2. Dünnschliff aus dem (B)-Horizont einer mitteleuropäischen Braunerde (Ungarn). Lockeres, hohlraumreiches, aus Mineralkörnern und gutgeflockten Schlämmstoffen bestehendes Schwammgefüge, das seine Grundmasse aus aneinanderhaftenden schorfig-krümeligen Aggregaten von brauner (7,5 YR 5/8) bis sepiabrauner (7,5 YR 4/4) Farbe bildet. Die Eisenhydroxydgele sind unbeweglich, Konkretionen werden kaum gebildet.

Abb. 3. B-Horizont einer fossilen gebleichten Parabraunerde (Lessivé) „der Kremser Bodenbildung “, Ziegelei Humlangen (Rottum) bei Laupheim (Württemberg). Der Dünnschliff zeigt ein geflocktes Braunerde-Grundgefüge aus Mineralkörnern und Schlämmstoffen, das von unbeweglichen Eisenhydroxydgelen sattbraun (7,5 YR 5/6) gefärbt ist und eine quer durch das Bild gehende dichte, hochbewegliche, sattocker (7,5 YR 6/8) gefärbte Fließplasmafüllung von Braunlehmcharakter in einer Leitbahn enthält. Die Aufnahme ist um $90^{2}-z u$ drehen, so daß die jetzige rechte Bildseite oben liegt.

Abb. 4. (B)-Horizont einer fossilen Braunerde, Erbbach, am Hohlweg z. Landhaus Marienhöhe, Rheingau. Weitgehend geflocktes lockeres Schwammgefüge, das durch die vorhandenen unbeweglichen Eisengele ockerbraun gefärbt ist. Die zahlreichen Hohlräume sind frei von Fließplasma. Vgl. das Präparat mit dem Standard-Gefüge einer Braunerde in Abb. 2. 

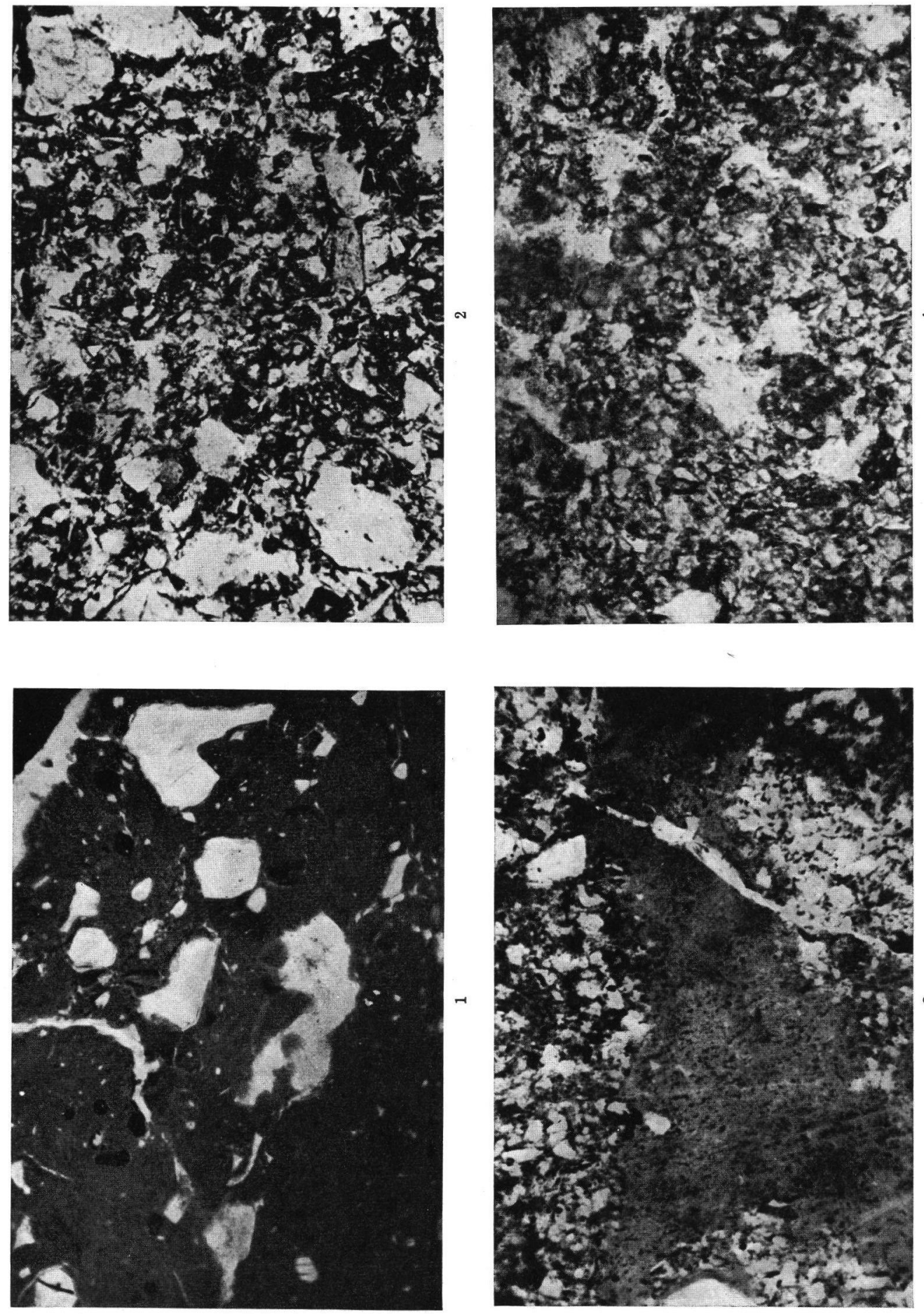
Abb. 1. A-Horizont einer fossilen gebleichten Parabraunerde der "Göttweiger Bodenbildung", Ziegelwerk Mühlacker, Schönenberg bei Heilbronn. Humoses, durchwaschenes und dichtgeschwämmtes Braunerdegefüge mit restlichen braunen Eisenhydroxydflocken. Braunlehm-Fließplasma fehlt bereits vorllständig, doch finden sich links oben noch kleine dunkelbraune Eisenhydroxydkonkretionen. Rechts unten Andeutung einer im Entstehen begriffenen, noch lockeren und hellbraun gefärbten Konkretion, die sich im Farbfilm stärker abhebt.

Abb. 2. Unterer, stärker durchwaschener Teil ( $A_{e}-$ Horizont) des A-Horizontes von Abb. 1 mit geringem Gehalt an restlichem Braunerde-Teilplasma. In der Bildmitte eine größere Eisenkonkretion von dunkelsepiabrauner Farbe (5 YR 3/2) umgeben von kleinen Konkretionen, welche die häufig auftretende, unregelmäßige, eigentümlich zerspratzte Form zeigen. Alle Anteile von Braunlehm-Teilplasma sind in den B-Horizont gewaschen.

Abb. 3. B-Horizont des gleichen Bodens. Dichtes, aber geflocktes Braunerde-Grundgefüge mit drei Leitbahn-Querschnitten, in dem sich wandständiges, sattocker gefärbtes (7,5 YR 5/8) Fließplasma von Braunlehmcharakter zeigt.

Abb. 4. B-Horizont des gleichen Bodens, gekreuzte Nicols. Längsschnitt durch eine schräg durch die Bildmitte laufende, sich nach unten verengende Leitbahn mit im oberen Teile wandständigem Fließplasma. Dieses zeigt Fließstrukturen und starke Teilchendoppelbrechung. Von der Bildmitte am oberen Rande nach rechts abwärts ziehen sich versprengte Reste von Fließplasma, die sich zufolge ihrer Teilchendoppelbrechung und ihren leuchtend goldgelben Polarisationsfarben im Farbfilm stark abheben. 

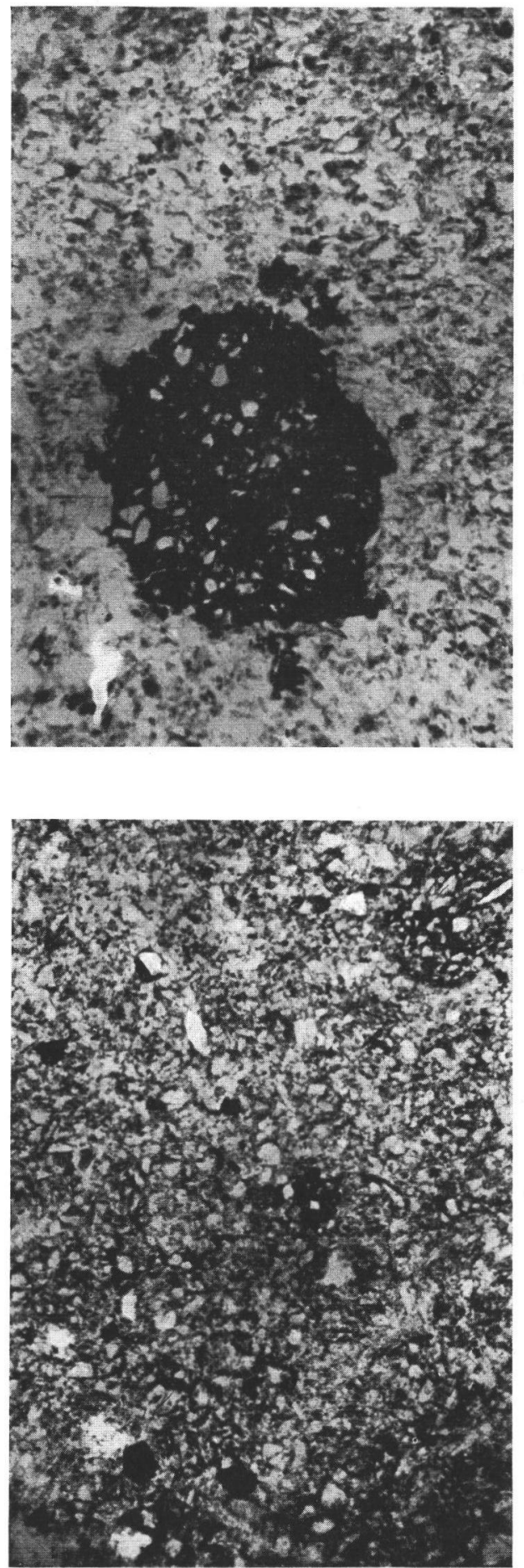
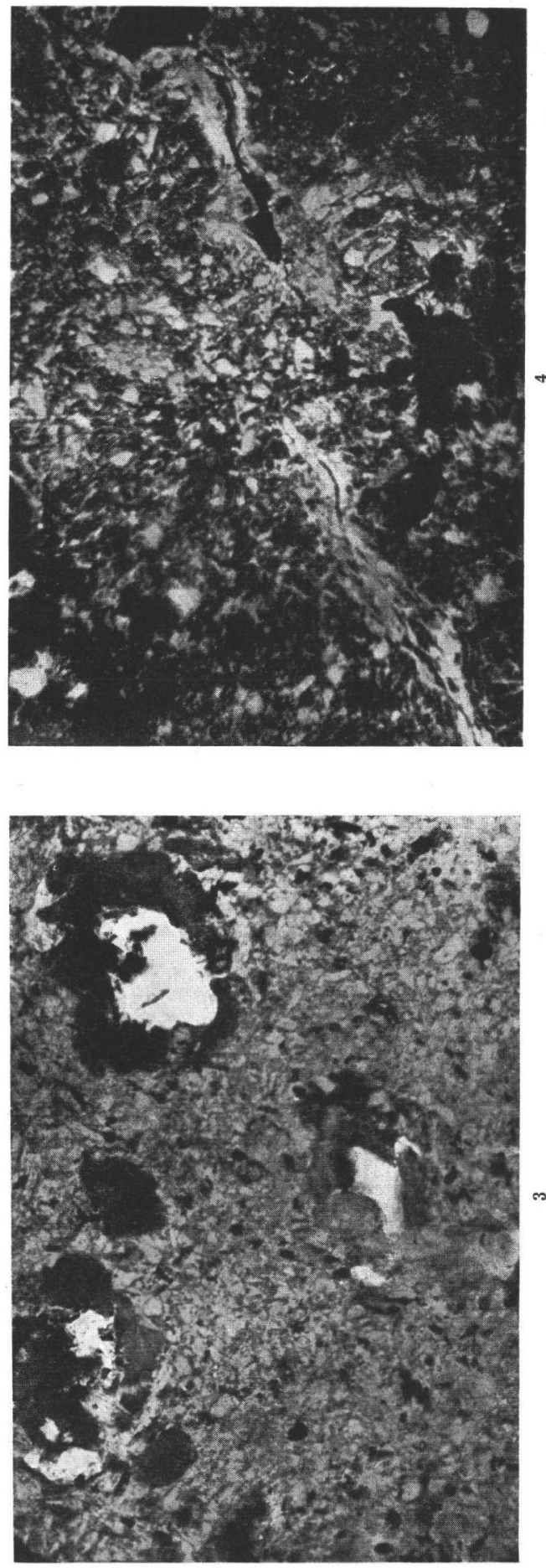
Abb. 1. B-Horizont des fossilen Lessivés von Laupheim („Kremser Bodenbildung“). Breite, mit Braunlehmplasma erfüllte, gekrümmte Leitbahn. An ihren Rändern das stark geflockte Braunerde-Grundgefüge.

Abb. 2. E/B-Horizont eines fossilen Lessivés der "Göttweiger Bodenbildung"; Westrand der Ziegelgrube Sindelfingen, Württemberg. In Fließerde verkneteter Teil einer leuchtend ockergelb (7,5 YR 6/8) gefärbten ehemaligen Leitbahnfüllung von Braunlehmplasma, in dem sich eine durch Fließbewegung in noch weichem teigigem Zustande verzogene und $\mathrm{z}$. T. zerrissene, tiefbraune (5 YR 2/3) Eisenkonkretion zeigt. Die Risse in ihrem Innern deuten an, daß sie noch weiterhin im Zerfall begriffen gewesen war.

Abb. 3. Dünnschliff des Braunlehmplasmas, das eine bis zu $10 \mathrm{~mm}$ breite Randspalte an einem Eiskeil völlig ausgefüllt. E/B-Material eines fossilen Lessivés der "Göttweiger Bodenbildung“. Ziegelwerk Mühlacker, Schönenberg bei Heilbronn. Das Bildfeld ist ganz von dichtem, braunocker (5 YR 5/8) gefärbtem Braunlehmplasma erfüllt. In ihm befinden sich fünf sepiabraune (7,5 YR 4/4) Gelflecke von Eisenhydroxyd mit diffusen Rändern. Die zahlreichen Sprungrisse in der lackartigen Masse sind von dunkelsepiabraunem Eisenhydroxydgel ausgeheilt.

Abb. 4. (B)-Horizont einer fossilen Parabraunerde auf jüngerem Deckenschotter, überdeckt von Rißmoräne. Umgebung von Ulm. Das leicht geflockte Grundgefüge ist von hellockerfarbigem (10 YR 8/8) Braunlehm-Plasma durchsetzt. In der Mitte eine konkretionsähnliche Ansammlung von Eisenhydroydgelen von dunkelbrauner Farbe (7,5 YR 3/2). 

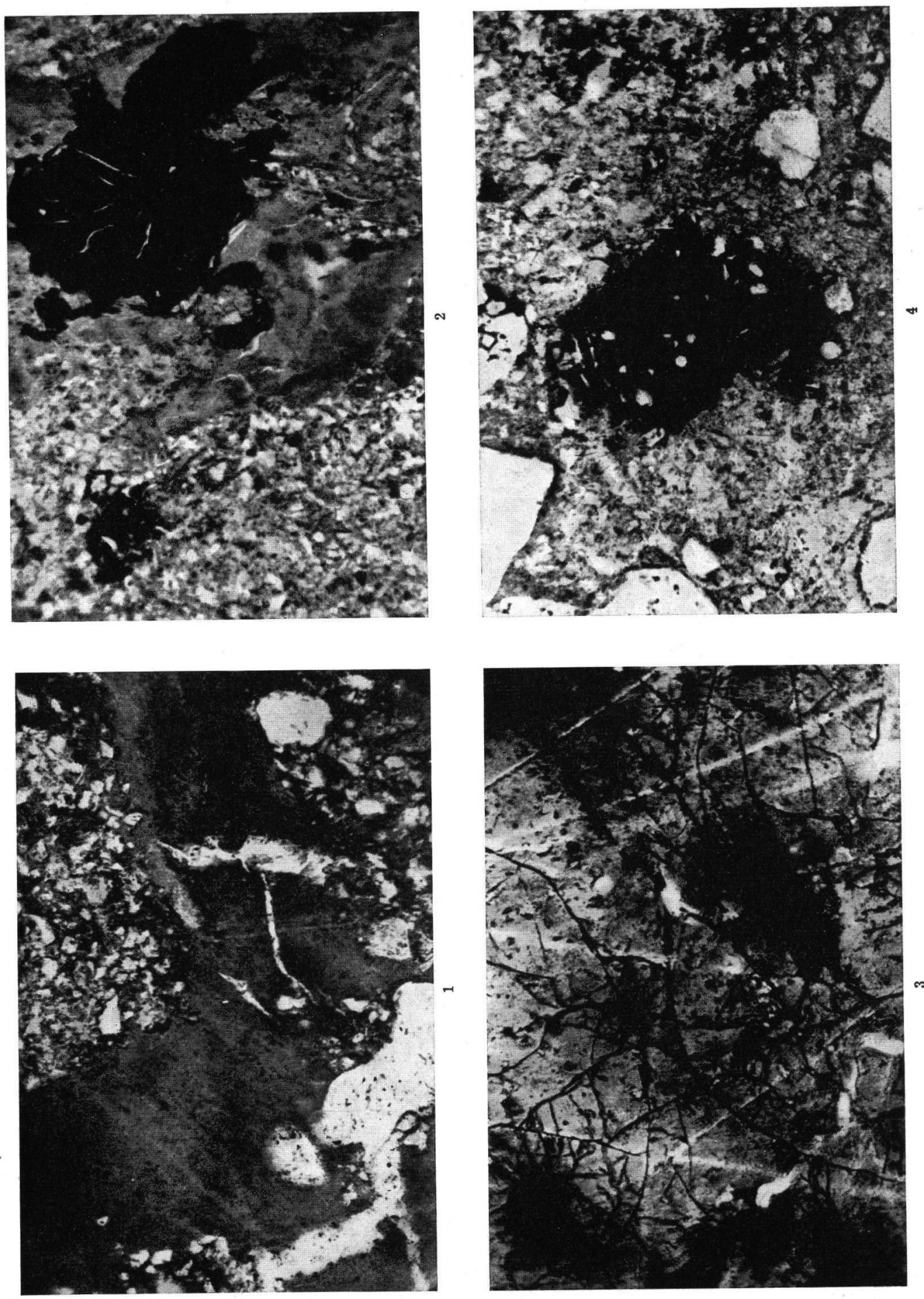


\section{Tafel V}

Abb. 1. Humushorizont eines fossilen, noch etwas anmoorigen Pseudo-Tschernosems. Umgebung von Stuttgart. Dichte, unstabile, humose Grundmasse von schwärzlichbrauner Farbe, in der sich humoses Fließplasma in unregelmäßigen Fleckchen vorfindet. Die Bodenmasse schwindet beim Austrocknen ungewöhnlich stark und bildet breite Sprungrisse.

Abb. 2. (B)-Horizont des fossilen Pseudo-Tschernosems, Moosinning, Bayern. Braunerdeähnliches, doch sehr dichtes Grundgefüge, das geflockte braune Schlämmstoffe enthält, durchsetzt von Braunlehmplasma, z. T. in Flecken z. T. in zahlreichen, zwiebelschalig aufblätternden bis guirlandenförmigen Leitbahnfüllungen von ockerbrauner Farbe (7,5 YR 5/8). Der Boden zeigt ein ähnliches Parabraunerde-Gefüge auch im A-Horizont, wobei die zahlreichen Leitbahnen humoses dunkelockergraues (7,5 YR 4/2) Braunlehm-Teilplasma führen.

Abb. 3. A-Horizont eines echten mittleren Tschernosems auf Löß, Nordbulgarien. Das humose Gefügeplasma setzt sich aus stark geflockten, dunkelbraungrauen Schlämmstoffen zusammen, die Bodenmasse zeigt ein lockeres, von zahlreichen Hohlräumen durchsetztes Schwammgefüge, die völlig frei von Einlagerungen eines Braunlehm-Teilplasmas sind, das hier vollständig fehlt.

Abb.4. G-Horizont eines Stagnogleys, Fleckenzone, Naßgalle im Leinaforst. Reduktion von Ferrieisen und gleichzeitige Eisenverarmung in den hellen Gefügepartien, Verdichtung in großen, dunkelsepiabraunen (7,5 YR 3/2), am Rande stark angefresseneen, sattbraun (7,5 YR 5/6) aufhellenden Eisenflecken. 



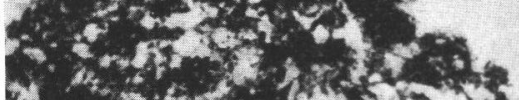

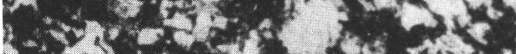

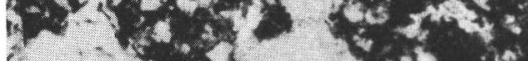

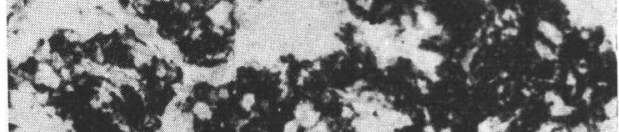

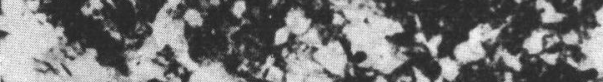

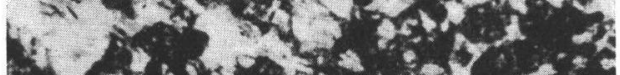

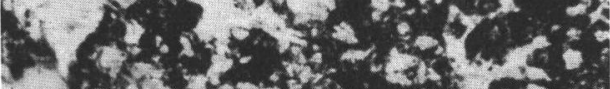

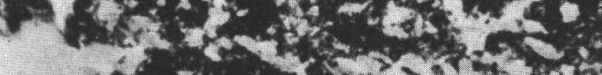

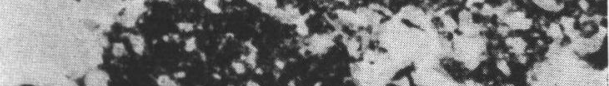
(x), $>1$ 
